\title{
Selective Use of Face Gesture Interface and Instrument Tracking System for Control of a Robotic Laparoscope Positioner
}

\author{
Atsushi Nishikawa ${ }^{1}$, Shuichi Asano ${ }^{1}$, Ryo Fujita $^{1}$, Satoshi Yamaguchi ${ }^{1}$, \\ Takahiro Yohda ${ }^{1}$, Fumio Miyazaki ${ }^{1}$, Mitsugu Sekimoto ${ }^{2}$, Masayoshi Yasui $^{2}$, \\ Yasuhiro Miyake ${ }^{2}$, Shuji Takiguchi ${ }^{2}$, and Morito Monden ${ }^{2}$ \\ 1 Department of Mechanical Science and Bioengineering, Graduate School of \\ Engineering Science, Osaka University, Toyonaka 560-8531, Japan \\ 2 Department of Surgery and Clinical Oncology, Osaka University \\ Graduate School of Medicine, Suita 565-0871, Japan
}

\section{Introduction}

Robotic camera positioners are now accepted and expected as assisting devices for solo surgery among endoscopic surgeons. However, camera guidance is an additional task for the operating surgeon, which may result in frustration, fatigue and longer surgery time. To cope with this problem, automatic camera guidance based on the visual tracking of surgical instruments may be effective, although it does not always provide an optimal view. We investigated the effectiveness of the selective use of automatic/manual control of a robotic laparoscope positioner.

\section{Materials and Methods}

We worked out two methods for controlling a robotic laparoscope positioner: face gesture interface [1] and instrument tracking system [2. The face gesture interface recognizes the surgeon's face gesture by processing the face images captured through a CCD camera. Using this gesture interface, the surgeon himself/herself can control the robot manipulator just as he/she wants. The instrument tracking system is based on the positional information of surgical instruments in the image of laparoscopic camera. The system first extracts instrument-attached markers from the laparoscope image and calculates the distance between the centroid of the markers and the center of the image. Then the system controls the robot manipulator automatically such that the distance may be reduced. Finally we combined these two controllers [3] and made a prototype system in which the surgeon can switch over the one to the other by stepping on a foot pedal.

\section{Results and Discussion}

An endoscopic surgeon used the prototype system to perform a laparoscopic cholecystectomy simulation with integrated pig organs (liver and gallbladder). 
The number of the experiments was two. The results are summarized as Case 1 and Case 2 in Table 1.

The difficulty of dissection of gallbladder(duct) from liver depends on its linkage condition. In order to carry out the detailed and complicated operation, fine adjustment of laparoscopic operating field is required. In such cases, manual camera control through the face gesture interface was essential, although the result of Case 1(b) suggests the surgeon may be able to dissect the gallbladder duct without the manual camera operation. In order to utilize the instrument tracking system, at least one instrument must be within the visual field, which limits its usability. Thus, the face gesture interface was also more effective in centering the target in the video image. Although the situation in which the instrument tracking was useful was limited, it greatly contributed to reduction of the surgeon's physical stress in camera guidance task.

Table 1. The results of laparoscopic cholecystectomy simulation. GB indicates gallbladder. "Manual by FG" and "Auto by IT" mean "Manual camera control by Face Gesture interface" and "Automatic camera control by Instrument Tracking."

\begin{tabular}{|c|c|c|c|c|c|c|}
\hline & \multicolumn{3}{|c|}{ Case 1 (unit: seconds) } & \multicolumn{3}{|c|}{ Case 2 (unit: seconds) } \\
\hline & \multirow[b]{2}{*}{ total time } & \multicolumn{2}{|c|}{ camera operation } & \multirow[b]{2}{*}{ total time } & \multicolumn{2}{|c|}{ camera operation } \\
\hline & & $\begin{array}{c}\text { Manual } \\
\text { by FG }\end{array}$ & $\begin{array}{l}\text { Auto } \\
\text { by IT }\end{array}$ & & $\begin{array}{c}\text { Manual } \\
\text { by FG }\end{array}$ & $\begin{array}{l}\text { Auto } \\
\text { by IT }\end{array}$ \\
\hline (a) centering GB duct & 106 & 11 & 10 & 38 & 9 & 0 \\
\hline (b) dissection of GB duct & 364 & 0 & 30 & 1120 & 30 & 23 \\
\hline (c) centering GB & 45 & 13 & 4 & 28 & 22 & 5 \\
\hline (d) dissection of GB & 988 & 47 & 47 & 710 & 72 & 38 \\
\hline$(\mathrm{a})+(\mathrm{b})+(\mathrm{c})+(\mathrm{d})$ & 1503 & 71 & 91 & 1896 & 133 & 66 \\
\hline
\end{tabular}

Acknowledgements. This work was supported by the Mikiya Science and Technology Foundation.

\section{References}

1. Nishikawa, A. et al. : Real-Time Visual Tracking of the Surgeon's Face for Laparoscopic Surgery. Medical Image Computing and Computer-Assisted InterventionMICCAI 2001, Proc. of 4th International Conference, Springer-Verlag (2001) 9-16

2. Nishikawa, A. et al.: Robust Visual Tracking of Multiple Surgical Instruments for Laparoscopic Surgery. CARS 2003, Computer Assisted Radiology and Surgery, Proc. of 17th International Congress and Exhibition, Elsevier Science (2003) 1372

3. Nishikawa, A. et al.: A Laparoscope Positioning System based on the Real-time Visual Tracking of Surgeon's Face and Surgical Instruments. CARS 2002, Computer Assisted Radiology and Surgery, Proc. of 16th International Congress and Exhibition, Springer-Verlag (2002) 1090 